\title{
An Approach to Analysis of Waveguide Arrays with Shaped Dielectric Inserts and Protrusions
}

\author{
Marat Davidovitz, Senior Member, IEEE
}

\begin{abstract}
The classical moment method solution of the waveguide-array problem is extended to allow for generally shaped dielectric matching inserts in the waveguide-to-free-space transition region. The aperture electric field is represented in terms of waveguide modes. To account for the presence of the matching inserts, the aperture fields are numerically propagated through the dielectric regions. Novel matching configurations, which extend the scanning range of waveguide elements or can be used to shape the element pattern in limited-scan applications, are proposed and analyzed.
\end{abstract}

Index Terms-Matching, phased array, waveguide.

\section{INTRODUCTION}

$\mathbf{T}$ HE related problems of waveguide phased-array matching and element pattern synthesis have been the subject of considerable research. Many practical approaches for dealing with the problem have been introduced and theoretical methods to analyze these have been developed. A partial account of this area of research can be found in [1]. Of particular interest here are efforts to address the matching problem by including dielectric inserts-protrusions in the waveguide-to-free-space transition region. Among them is the early analytical work by Lewis et al. [2], [3] on protruding dielectric slabs. In the Russian literature, analogous contemporaneous developments are cited in the paper by Skobelev et al. [4], which presents a numerical method for dealing with shaped protruding dielectric elements. The formulation described by Skobelev et al. appears to be suitable for two-dimensional (2-D) scalar problems in which the feeding waveguide is uniformly filled. In many situations of practical interest, the dielectric intruding into the guide may be shaped [6] and a general approach is needed to analyze dielectric matching transformers, which intrude/protrude into both the waveguide and free space. The purpose of this paper is to present a methodology that can uniformly deal with both the waveguide and free-space portions of the problem and is ultimately applicable to three-dimensional (3-D), as well as 2-D, geometries.

\section{FORMULATION}

A graphical statement of the problem is presented in Fig. 1. The configuration is a periodic structure of open-ended parallel-plate waveguides (PPWs) of height $b$ spaced a distance

Manuscript received October 11, 1999. This work was supported by the Air Force Office of Scientific Research Directorate of Academic and International Affairs under the Window on Europe Program.

The author is with the Air Force Research Laboratory/SNHA, Hanscom AFB, MA 01731-3010 USA.

Publisher Item Identifier S 0018-9480(01)01077-8.

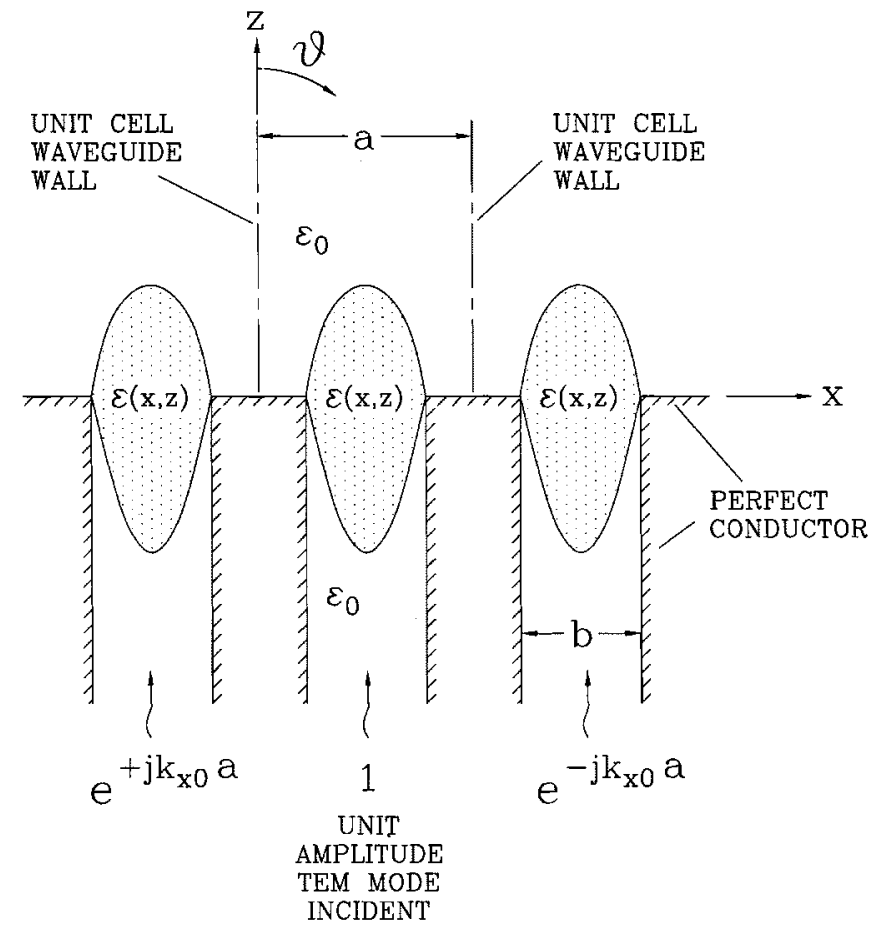

Fig. 1. Periodic array of parallel-plate waveguides with a general dielectric distribution in the waveguide-to-free-space transition region.

$a$ apart. The elements are excited with a constant progressive phase increment $k_{x 0} a=k_{0} a \sin \theta$ between adjacent unit-magnitude sources, where $k_{0}=\omega \sqrt{\varepsilon_{0} \mu_{0}}$ is the free-space propagation constant and $\theta$ is the scan angle measured from broadside. A generally shaped dielectric distribution is included in the waveguide-to-space transition regions. The problem is 2-D (independent of the $y$-coordinate) and the fields are taken to be $\mathrm{TE}_{y}$ to simulate $E$-plane scan conditions. This commonly used 2-D analog possesses the most important physical attributes of the more practical 3-D problem [3]. Moreover, the 2-D formulation is algebraically simpler and, therefore, permits a clearer presentation of its analytical and numerical properties. It should also be noted that of the two possible 2-D formulations, namely, $E$ - and $H$-planes, the former is expected to have more severe convergence requirements due to the discontinuous nature of the normal electric-field component at dielectric-air interfaces.

The parameters of interest for the stated problem will be obtained by analyzing a single unit cell of the underlying periodic structure, shown in Fig. 2. It is assumed throughout that the dielectric inhomogeneity is confined to a finite interval $\left[z_{b}, z_{e}\right]$ along the $z$-axis. Following the standard moment method approach, the problem volume is decomposed into two regions connected by the waveguide aperture $A \equiv\left\{\left(x \in\left[x_{w}, x_{u}\right], z=\right.\right.$ 


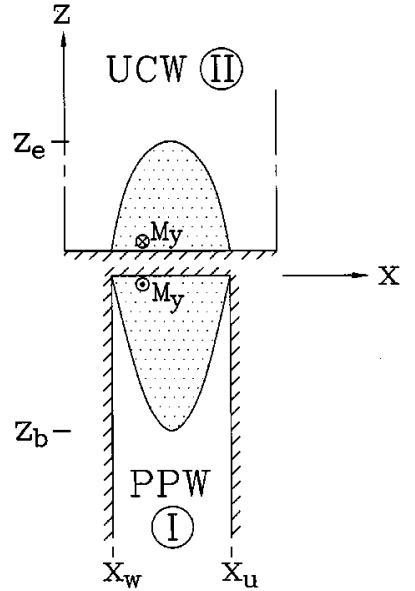

Fig. 2. Unit cell of the array decomposed into two regions to facilitate a moment method formulation.

$0)\}$. Roman numerals I and II are used to identify quantities associated with the PPW and the unit-cell waveguide (UCW) volumes, respectively. Placement of oppositely directed equivalent magnetic currents on both sides of a perfect electric conductor (PEC) screen stretching across the aperture ensures the continuity of the total tangential electric field $E_{A} \equiv E_{x}[(x, z) \in A]$, while imposition of the same condition on the total magnetic field yields the following formal integral equation statement:

$$
\begin{aligned}
& \qquad \begin{array}{l}
H_{y s}^{I}+H_{y}^{I}\left(M_{y}\right)=H_{y}^{I I}\left(-M_{y}\right), \quad(x, z) \in A \\
\begin{array}{l}
M_{y} \\
H_{y}^{I}\left(M_{y}\right) \quad \text { equivalent aperture magnetic current; } \\
\text { magnetic field excited in the PPW by the } \\
\text { source } M_{y} \text { in the presence of the dielectric, } \\
\text { with the aperture covered with a PEC; } \\
\text { magnetic field excited in the UCW by the } \\
\text { source }-M_{y} \text { in the presence of the dielectric, } \\
\text { with the aperture covered with a PEC; } \\
\text { source magnetic field excited in the PPW by } \\
\text { the incident TEM mode } \text { in the presence of the } \\
\text { dielectric, with the aperture covered with a }
\end{array} \\
\begin{array}{l}
\text { PEC. } \\
H_{y}^{I I}\left(-M_{y s}^{I}\right.
\end{array} \\
\text { After introducing a basis set }\left\{M_{j}, j=1,2, \ldots, N_{B F}\right\} \text { and } \\
\text { the expansion } M_{y}=\sum_{j=1}^{N_{B F}} V_{j} M_{j}, \text { where } V_{j} \text { are to be de- } \\
\text { termined, the approximate solution to }(1) \text { is obtained by the } \\
\text { Galerkin technique [5]. The resulting set of linear equations can } \\
\text { be written as }
\end{array} \\
& \sum_{j=1}^{N_{B F}} V_{j} Y_{i j}^{I}+\sum_{j=1}^{N_{B F}} V_{j} Y_{i j}^{I I}=I_{s i}, \quad i=1,2, \ldots, N_{B F}
\end{aligned}
$$

where the following definitions apply:

$$
\begin{aligned}
Y_{i j}^{I} & \equiv-\left\langle M_{i}, H_{y}^{I}\left(M_{j}\right)\right\rangle \\
Y_{i j}^{I I} & \equiv\left\langle M_{i}, H_{y}^{I I}\left(-M_{j}\right)\right\rangle \\
& =-\left\langle M_{i}, H_{y}^{I I}\left(M_{j}\right)\right\rangle \\
I_{s i} & \equiv\left\langle M_{i}, H_{y s}^{I}\right\rangle \\
\langle P, Q\rangle & =\int_{A} P Q d x .
\end{aligned}
$$

Thus far, the steps taken toward the solution of the problem have been conventional. Moreover, if the configuration at hand were reduced to the two commonly encountered cases, namely, those in which the PPW and/or UCW were filled uniformly or with $x$-invariant stratified dielectrics, the solution could proceed along the usual path. Briefly, this would entail the derivation of Green functions for the two regions, using the eigenspectra of transverse modes, followed by the evaluation of the magnetic fields $H_{y}^{I, I I}\left(M_{j}\right)$ as convolutions of the sources with the respective Green function and concluded with the field testing in (3). In principle, this approach can be extended to more general dielectric distributions, as was done in [3], by deriving eigenmodes for nonuniformly filled UCW/PPW cross sections. However, in all but the most basic 2-D configurations, the eigenmode problem itself is very challenging and intensive, particularly when the filled waveguide cross sections vary as functions of the propagation axis variable $z$. An alternate, more direct, and practicable method of evaluating $H_{y}^{I, I I}\left(M_{j}\right)$ is proposed and studied here. The analysis will be carried out in detail for Region II (UCW). The derivation for the PPW is completely analogous and will be given minimal discussion. For convenience and clarity, the region identifier will be suppressed during the following derivation.

The mathematical relations governing fields excited by the source $M_{j}$ in Region II are

$$
\begin{aligned}
\frac{\partial E_{x}(x, z)}{\partial z}-\frac{\partial E_{z}(x, z)}{\partial x} & =-j \omega \mu_{0} H_{y}(x, z) \\
\frac{\partial H_{y}(x, z)}{\partial x} & =j \omega \epsilon(x, z) E_{z}(x, z) \\
-\frac{\partial H_{y}(x, z)}{\partial z} & =j \omega \epsilon(x, z) E_{x}(x, z)
\end{aligned}
$$

subject to the boundary conditions

$$
\begin{aligned}
F(x+a, z) & =F(x, z) e^{-j k_{x 0} a} \\
F & \equiv E_{x}, E_{z}, H_{y} \\
E_{x}(x, 0) & = \begin{cases}-M_{j}(x), & (x, z) \in A \\
0, & (x, z) \notin A\end{cases}
\end{aligned}
$$

with the addition of the radiation condition for $z \rightarrow \infty$. Note, anticipating subsequent numerical developments, the surface magnetic current source was incorporated into the mathematical formulation as a boundary condition.

The problem presented in (5) will be solved by adapting and generalizing, to include sources and UCW, a technique used to evaluate reflection/transmission characteristics of dielectric discontinuities in waveguides. Proposed by Schelkunoff [7] and subsequently elaborated in, among others, [8], the technique makes use of the empty waveguide mode spectrum as a basis for representing the transverse variation of the fields in the inhomogeneously filled waveguide. The field dependence on the longitudinal variable generally must be determined numerically.

Following the prescription, the Floquet modes of the air-filed UCW are introduced first:

$$
\begin{aligned}
H_{y \sigma n}(x, z) & =h_{y \sigma n}(x) e^{-j \beta_{\sigma n} z} \\
& =\frac{1}{\sqrt{a}} e^{-j k_{x n} x} e^{-j \beta_{\sigma n} z} \\
E_{x \sigma n}(x, z) & =e_{x \sigma n}(x) e^{-j \beta_{\sigma n} z} \\
& =Z_{\sigma n} h_{y \sigma n}(x) e^{-j \beta_{\sigma n} z}
\end{aligned}
$$




$$
\begin{aligned}
E_{z \sigma n}(x, z) & =e_{z \sigma n}(x) e^{-j \beta_{\sigma n} z} \\
& =\frac{1}{j \omega \varepsilon_{0}} \frac{\partial h_{y \sigma n}}{\partial x}(x) e^{-j \beta_{\sigma n} z} \\
k_{x n} & =\frac{2 \pi n}{a}+k_{x 0}, \quad n=0, \pm 1, \pm 2, \ldots ; \\
\beta_{+n} & =\sqrt{k_{0}^{2}-k_{x n}^{2}} \\
& =\frac{-\beta_{-n}}{\beta_{\sigma n}} \\
Z_{\sigma n} & \equiv \frac{\beta_{\sigma n}}{\omega \varepsilon_{0}} .
\end{aligned}
$$

$$
\begin{aligned}
J_{\nu m}^{\sigma n}(z) & =\int_{0}^{a} d x h_{y \sigma n}(x) h_{y \nu m}^{\dagger}(x) \\
K_{\nu m}^{\sigma n}(z) & =\int_{0}^{a} d x \varepsilon_{r}(x, z) e_{x \sigma n}(x) e_{x \nu m}^{\dagger}(x)
\end{aligned}
$$

The reduction of the formulation in (5) is completed with the addition of the necessary boundary conditions. First, it should be noted the Floquet-periodicity requirement in (5d) is naturally satisfied through the choice of the Floquet mode basis for field representation. The boundary condition in $(5 \mathrm{e})$ and the radiation condition for $z \longrightarrow \infty$ are addressed next. To reduce the former of these to the proper form, a series of steps, analogous to those used to obtain (10), is carried out. The steps are as follows.

Step 1) Substitution of (8b) into (5e).

Step 2) Multiplication of the result by $h_{y \nu m}^{\dagger}(x)$.

Step 3) Integration over the UCW cross section and application of (7b).

$$
\int_{0}^{a} d x e_{x \sigma n}(x) h_{y \nu m}^{\dagger}(x)=Z_{\sigma n} \delta_{m n}
$$

where the dagger " $\nmid$ " denotes adjoint quantities, e.g., $e_{x \nu m}^{\dagger} \equiv$ $\left[e_{x \nu m}\left(\beta_{\nu m} \rightarrow \beta_{\nu m}^{*}\right)\right]^{*}$, and $\delta_{m n}$ is the Kronecker symbol.

Let the transverse-to- $z$-field components in (5) be expanded in a sum of Floquet harmonics with unknown coefficients $P_{\sigma n}(z)$ as

$$
\begin{aligned}
& H_{y}(x, z)=\sum_{\sigma, n} P_{\sigma n}(z) h_{y \sigma n}(x) \\
& E_{x}(x, z)=\sum_{\sigma, n} P_{\sigma n}(z) e_{x \sigma n}(x)
\end{aligned}
$$

where it follows via (5b), (6c), and the definition $\epsilon_{r}(x, z) \equiv$ $\epsilon(x, z) / \varepsilon_{0}$ that

$$
E_{z}(x, z)=\frac{1}{\epsilon_{r}(x, z)} \sum_{\sigma, n} P_{\sigma n}(z) e_{z \sigma n}(x)
$$

The major step in the derivation requires several straightforward, but cumbersome, algebraic manipulations, namely,

- substitution of (8) and (9) into (5a) and (5c);

- multiplication of (5a) and (5c) by $h_{y \nu m}^{\dagger}(x)$ and $e_{x \nu m}^{\dagger}(x)$, respectively;

- summation of the two equations;

- integration of the result over the UCW cross section and application of (7a).

These procedures reduce the problem to a coupled set of ordinary differential equations (ODEs), with the longitudinal coordinate $z$ as the independent variable

$$
\begin{aligned}
2 Z_{\nu m} P_{\nu m}^{\prime}(z) & \\
=\sum_{\sigma, n} P_{\sigma n}(z) & {\left[\frac{1}{j \omega \varepsilon_{0}} I_{\nu m}^{\sigma n}(z)-j \omega \mu_{0} J_{\nu m}^{\sigma n}(z)\right.} \\
& \left.-j \omega \varepsilon_{0} K_{\nu m}^{\sigma n}(z)\right]
\end{aligned}
$$

where $\sigma, \nu \in\{+,-\}, m, n \in\{0, \pm 1, \pm 2, \ldots\}$, prime indicates differentiation with respect to $z$, and the newly introduced quantities are defined as follows:

$$
I_{\nu m}^{\sigma n}(z)=\int_{0}^{a} d x \frac{\partial}{\partial x}\left[\frac{1}{\varepsilon_{r}(x, z)} \frac{\partial h_{y \sigma n}}{\partial x}(x)\right] h_{y \nu m}^{\dagger}(x)
$$

The resulting expressions are written as follows:

$$
\begin{aligned}
{\left[P_{+m}(0)-P_{-m}(0)\right] Z_{+m} } & =-L_{m}, \quad m \in\{0, \pm 1, \pm 2, \ldots\} \\
L_{m} & =\left\langle M_{j}(x), h_{y \nu m}^{\dagger}(x)\right\rangle .
\end{aligned}
$$

Finally, since the fields are expressed in terms of incoming and outgoing waves traveling along the $z$-direction, the radiation condition takes on a particularly simple form

$$
P_{-m}\left(z_{e}\right)=0, \quad m \in\{0, \pm 1, \pm 2, \ldots\}
$$

where it is recalled $z=z_{e}$ is the location beyond which the inhomogeneity disappears and, therefore, no reflected ( $-z$-going) waves are generated.

The ODE set (10), (12), and (14) taken together constitutes a well-posed two-point boundary value problem for the unknown coefficients $P_{\sigma n}(z)$. In general, this problem can be solved by numerical means only. Under certain condition, e.g., weak coupling, asymptotic techniques may be applicable.

An analogous derivation has been performed to find the field $H_{y}^{I}\left(M_{j}\right)$ in Region I (PPW). To introduce the notation for future reference, let the modes of homogeneously filled PPW be given by

$$
\begin{array}{rl}
H_{y \sigma n}(x, z) & =\mathrm{h}_{y \sigma n}(x) e^{-j \xi_{\sigma n} z} \\
& =\sqrt{\frac{2-\delta_{0 n}}{b}} \cos \left[\frac{n \pi}{b}\left(x-x_{w}\right)\right] e^{-j \xi_{\sigma n} z} \\
E_{x \sigma n}(x, z) & =\mathrm{e}_{x \sigma n}(x) e^{-j \xi_{\sigma n} z} \\
& =\mathrm{Z}_{\sigma n} \mathrm{~h}_{y \sigma n}(x) e^{-j \xi_{\sigma n} z} \\
E_{z \sigma n}(x, z) & =\mathrm{e}_{z \sigma n}(x) e^{-j \xi_{\sigma n} z} \\
& =\frac{1}{j \omega \varepsilon_{0}} \frac{\partial \mathrm{h}_{y \sigma n}}{\partial x}(x) e^{-j \xi_{\sigma n} z} \\
x_{w} \leq x \leq x_{w}+b=x_{u} & n=0,1,2, \ldots ; \sigma=+,- \\
\xi_{+n} & =\sqrt{k_{0}^{2}-\left(\frac{n \pi}{b}\right)^{2}} \\
& =\frac{-\xi_{-n}}{\xi_{\sigma n}} \\
\mathrm{Z}_{\sigma n} & \equiv \frac{\xi_{\sigma n}}{\omega \varepsilon_{0}}
\end{array}
$$


The fields excited in the PPW containing a dielectric inhomogeneity by a source $M_{j}$ can be expressed as follows:

$$
\begin{aligned}
& H_{y}(x, z)=\sum_{\sigma, n} Q_{\sigma n}(z) \mathrm{h}_{y \sigma n}(x) \\
& E_{x}(x, z)=\sum_{\sigma, n} Q_{\sigma n}(z) \mathbf{e}_{x \sigma n}(x) . \\
& E_{z}(x, z)=\frac{1}{\epsilon_{r}(x, z)} \sum_{\sigma, n} Q_{\sigma n}(z) \mathbf{e}_{z \sigma n}(x) .
\end{aligned}
$$

The ODE set for the coefficients $Q_{\sigma n}(z)$ is formally obtained from (10) and (11) via the substitutions $\left\{P, \beta_{\sigma n}, Z_{\sigma n}, e_{x \sigma n}, h_{y \sigma n}\right\} \longrightarrow\left\{Q, \xi_{\sigma n}, \mathrm{z}_{\sigma n}, \mathrm{e}_{x \sigma n}, \mathrm{~h}_{y \sigma n}\right\}$. The boundary conditions are obtained using the same substitution with two additional minor modifications, namely, the omission of the minus sign in front of $L_{m}$ in (12) and the replacement of the radiation condition (14) by $Q_{+m}\left(z_{b}\right)=0$, where it is assumed that the inhomogeneity starts at $z=z_{b}$, as indicated in Fig. 2.

The evaluation of the source field term $H_{y s}^{I}$, defined in (1), is needed in order to complete the derivation. Let the unit-amplitude TEM mode, distinguished by the index " 0 ," be incident in the PPW. The source fields expansions are formally identical to (17), i.e.,

$$
H_{s y}(x, z)=\sum_{\sigma, n} Q_{\sigma n}^{s}(z) \mathrm{h}_{y \sigma n}(x)
$$

etc., where the superscript on $Q_{\sigma n}^{s}$ refers to the source field. In accordance with the definition of the source field, the coefficients $Q_{\sigma n}^{s}$ are determined by the same ODE set as the field $H_{y}^{I}\left(M_{j}\right)$ with boundary condition replaced by

$$
\begin{aligned}
{\left[Q_{+m}^{s}(0)-Q_{-m}^{s}(0)\right] } & =0, \quad m \in\{0,1,2, \ldots\} \\
Q_{+m}^{s}\left(z_{b}\right) & =\delta_{m 0} .
\end{aligned}
$$

The solution is completed by returning to the moment method formulation and expressing (3) in terms of the derived field quantities. Thus,

$$
\begin{aligned}
H_{s y}^{I}(x, z) & =\sum_{\sigma, n} Q_{\sigma n}^{s}(z) \mathrm{h}_{y \sigma n}(x) \\
H_{y}^{I}\left(M_{j}\right) & =\sum_{\sigma, n} Q_{\sigma n}^{j}(z) \mathrm{h}_{y \sigma n}(x) \\
H_{y}^{I I}\left(M_{j}\right) & =\sum_{\sigma, n} P_{\sigma n}^{j}(z) h_{y \sigma n}(x)
\end{aligned}
$$

from which it follows:

$$
\begin{aligned}
I_{s i} & =\sum_{\sigma, n} Q_{\sigma n}^{s}(0) C_{n}^{i} \\
Y_{i j}^{I} & =-\sum_{\sigma, n} Q_{\sigma n}^{j}(0) C_{n}^{i} \\
Y_{i j}^{I I} & =-\sum_{\sigma, n} P_{\sigma n}^{j}(0) D_{n}^{i}
\end{aligned}
$$

with

$$
\begin{aligned}
C_{n}^{i} & \equiv\left\langle M_{i}, \mathrm{~h}_{y \sigma n}(x)\right\rangle \\
D_{n}^{i} & \equiv\left\langle M_{i}, h_{y \sigma n}(x)\right\rangle .
\end{aligned}
$$

After the linear system (2) is solved for the expansion coefficients $V_{j}$, the engineering descriptors of the antenna element, namely, the incident wave reflection coefficient $R$ and the ele- ment gain pattern $g(\theta)$ can be computed by means of the following formulas:

$$
\begin{aligned}
R\left(z_{b}\right) & =Q_{-0}^{s}\left(z_{b}\right)+\sum_{j=1}^{N_{B F}} V_{j} Q_{-0}^{j}\left(z_{b}\right) \\
g(\theta) & =\left|T_{0}(\theta)\right|^{2} \cos \theta
\end{aligned}
$$

where

$$
\left|T_{0}(\theta)\right|^{2}=\left\{\frac{Z_{+0}}{Z_{+0}}\left|\sum_{j=1}^{N_{B F}} V_{j} P_{+0}^{j}\left(z_{e}\right)\right|^{2}\right\}_{k_{x 0}=k_{0} \sin \theta .}
$$

\section{SPECIFIC EXAMPLES}

The formulation presented in the preceding section was completely general - applicable to arbitrary dielectric distributions. The theory will now be put to the test by application to several specific dielectric arrangements. The first two were previously analyzed by other techniques [3], [4] and are used here for purposes of theoretical verification.

Referring to Fig. 2, let the PPW be centered within the unit cell, such that $x_{w}=(a-b) / 2, x_{u}=(a+b) / 2$, and be completely filled with material of relative dielectric constant $\epsilon_{r}$. Let the protrusion into the UCW be made of the same material and be symmetrically distributed with respect to the UCW center line. The dielectric distribution may be defined as follows: $\epsilon(x, z)$

$$
= \begin{cases}\epsilon_{r} \epsilon_{0}, & x \in\left[x_{w}, x_{u}\right] ; z \in(-\infty, 0] \\ \epsilon_{r} \epsilon_{0}, & x \in[a / 2-p(z), a / 2+p(z)] ; z \in\left[0, z_{e}\right] \\ \epsilon_{0}, & x \notin[a / 2-p(z), a / 2+p(z)] ; z \in\left[0, z_{e}\right]\end{cases}
$$

where $p(z)(\leq a / 2)$ is a profile or shape function. The PPW modes will be used in all the forthcoming cases as the basis for the expansion of the aperture electric field, i.e.,

$$
\begin{array}{r}
M_{i}(x)=\sqrt{\frac{2-\delta_{0(i-1)}}{b}} \cos \left[\frac{(i-1) \pi}{b}\left(x-x_{w}\right)\right], \\
x \in\left[x_{w}, x_{u}\right], i \in\left\{1,2, \ldots, N_{B F}\right\} .
\end{array}
$$

Upon substituting (25) and (26) into the formulation and carrying out the calculus, the following set of equations are obtained:

$$
\begin{aligned}
\left(P_{\nu m}^{j}\right)^{\prime}(z) & =\sum_{\sigma, n} T_{\nu m}^{\sigma n}(z) P_{\sigma n}^{j}(z) \\
{\left[P_{+m}^{j}(0)-P_{-m}^{j}(0)\right] } & =\frac{1}{2 Z_{+m}}\left(D_{n}^{j}\right)^{*} \\
P_{-m}^{j}\left(z_{e}\right) & =0
\end{aligned}
$$

where

$$
\begin{aligned}
T_{\nu m}^{\sigma n}(z)= & -j \beta_{\nu m} \delta_{m n} \delta_{\nu \sigma} \\
& +f_{m n}(z)\left[j \frac{k_{x m} k_{x n}}{2 \beta_{\nu m}}\left(\frac{1}{\epsilon_{r}}-1\right)-j \frac{\beta_{\sigma n}}{2}\left(\epsilon_{r}-1\right)\right] \\
f_{m n}(z)= & (-1)^{n-m} \frac{\sin \left[\frac{2 \pi(n-m) p(z)}{a}\right]}{\pi(n-m)} \\
D_{n}^{i}= & -j \sqrt{\frac{2-\delta_{0 i}}{a b}} \frac{k_{x m}}{k_{x m}^{2}-\left(\frac{i \pi}{b}\right)^{2}} \\
& \cdot e^{-j k_{x m} a / 2}\left(e^{-j k_{x m} b / 2}-(-1)^{i} e^{j k_{x m} b / 2}\right)
\end{aligned}
$$




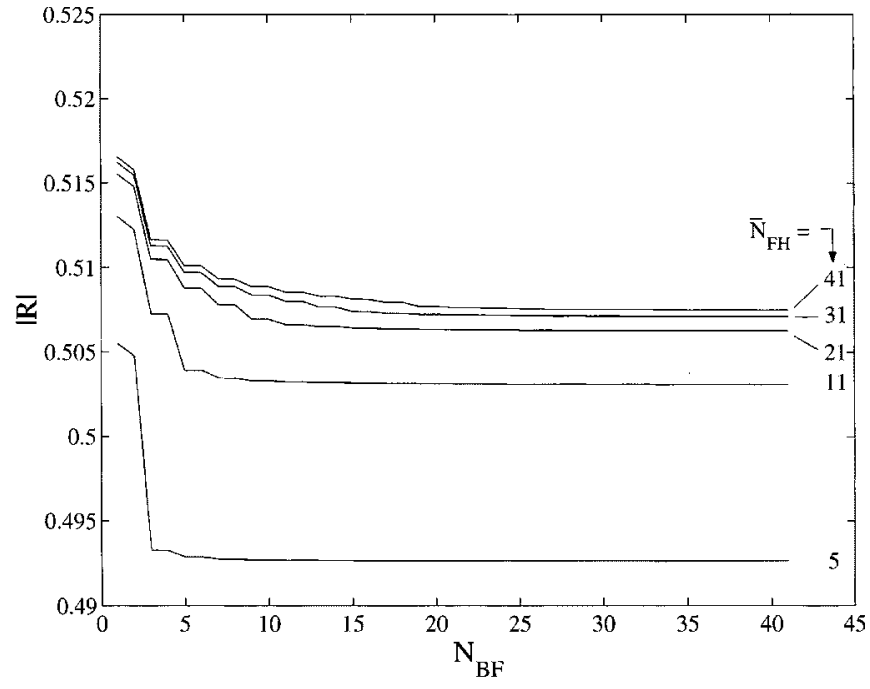

Fig. 3. Convergence study of the input reflection coefficient for a fixed scan angle $\left(\theta=5^{\circ}\right)$ as a function of the number of apertures expansion modes $\left(N_{B F}\right)$ and Floquet harmonics in the UCW region $\left(\bar{N}_{F H}\right)$. The unit cell parameters are [3, Fig. 3] $a / \lambda_{0}=0.535, b / a=0.432, \varepsilon_{r}=2.56, p(z)=$ $b / 2, z_{e}=\lambda_{0} / 2$.

and $\sigma, \nu \in\{+,-\}, m, n \in\left\{0, \pm 1, \pm 2, \ldots, \pm N_{F H}\right\}$. Note, the UCW mode spectrum has now been truncated at $\pm N_{F H}$ for computational purposes.

The moment method elements now become

$$
\begin{aligned}
I_{s i} & =2 \delta_{i 1} \\
Y_{i j}^{I} & =\mathrm{Y}_{+i} \delta_{i j} \\
Y_{i j}^{I I} & =\sum_{\sigma, n} P_{\sigma n}^{j}(0) D_{n}^{i}
\end{aligned}
$$

where $\mathrm{Y}_{+i}=1 / \mathrm{Z}_{+i}=\left(\omega \varepsilon_{0} \varepsilon_{r} / \xi_{+i}\right)$ and the reflection coefficient is simply

$$
R(0)=1-\frac{\omega \varepsilon_{0} \varepsilon_{r}}{\xi_{+0}} V_{1} .
$$

The example at hand will now be addressed from the numerical standpoint to ascertain the accuracy and convergence properties of the analytical method used. The data found in the cited literature will be used as standards of reference. Two inherent convergence parameters, namely, the number of aperture expansion functions/PPW modes $\left(N_{B F}\right)$ and the total number of Floquet (UCW) modes $\left(\bar{N}_{F H}=2 N_{F H}+1\right)$, will be controlled. The element configuration assumed for this purpose is that of $[3$, Fig. 3], with $a / \lambda_{0}=0.535, b / a=0.432, \varepsilon_{r}=2.56, p(z)=$ $b / 2, z_{e}=\lambda_{0} / 2$. The convergence behavior of the magnitude of the input reflection coefficient $|R|$ for a fixed scan angle $\left(\theta=5^{\circ}\right)$ as a function of $N_{B F}$, with $\bar{N}_{F H}$ serving as a parameter, is inferred from Fig. 3. It is observed that for a fixed $\bar{N}_{F H}$, $|R|$ relaxes to a constant value after a relatively small number of basis functions is included. On the other hand, if $N_{B F}$ is held constant, the changes in the reflection coefficient become practically negligible after the value $\bar{N}_{F H} \approx 25$ is exceeded. No pronounced relative convergence effects are observed. In Fig. 4 the gain pattern as a function of the scan angle $\theta$ for the same element is presented and compared with graphically obtained data from [3, Fig. 3]. Very good agreement is observed; likewise, for

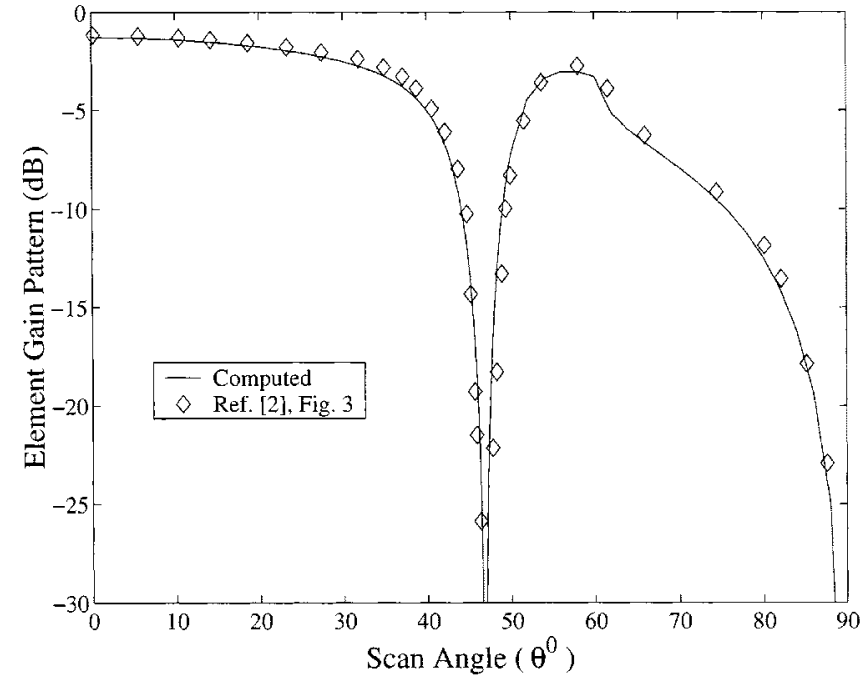

Fig. 4. Comparison of the element gain pattern computed for the element in Fig. 3 with data from [3, Fig 3].

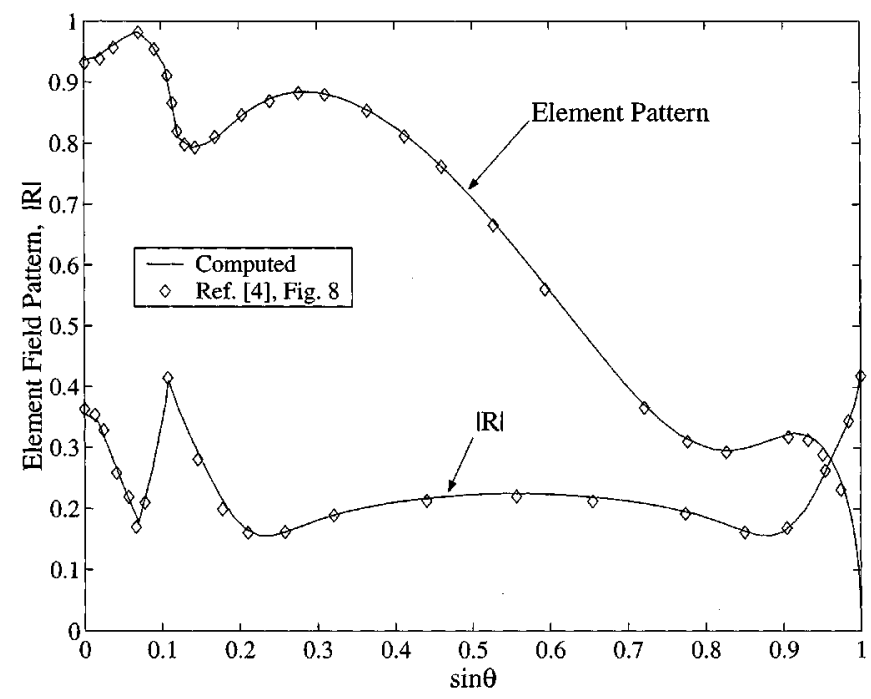

Fig. 5. Comparison of computed element field pattern and input reflection coefficient with data from [4, Fig. 8].

Fig. 5, which shows the computed results for the element analyzed in [4, Fig. 8] (the profile function for this element is cumbersome and is not reproduced here).

The computational properties of the presented method are largely determined by the numerical integration of the ODE set (10), (12), and (14). This operation was carried out using the IMSL 1 routine "BVPFD," which is based on a variable-order variable step-size finite-difference method with adaptive grid control. Consequently, the solution is highly robust and reliable, although the overhead associated with adaptive iterations slows it down somewhat. The program execution times for the data presented here are in the range of 20-35 s of CPU time per frequency/scan angle on a 266-MHz Pentium II machine.

It has been amply demonstrated that dielectric matching elements, be they sheets, as in [6] or slabs, analyzed in [3], when positioned close to the aperture, where fields are strongest, are apt to excite spurious trapped waves that have been directly

${ }^{1}$ Visual Numerics Inc., Houston, TX. 

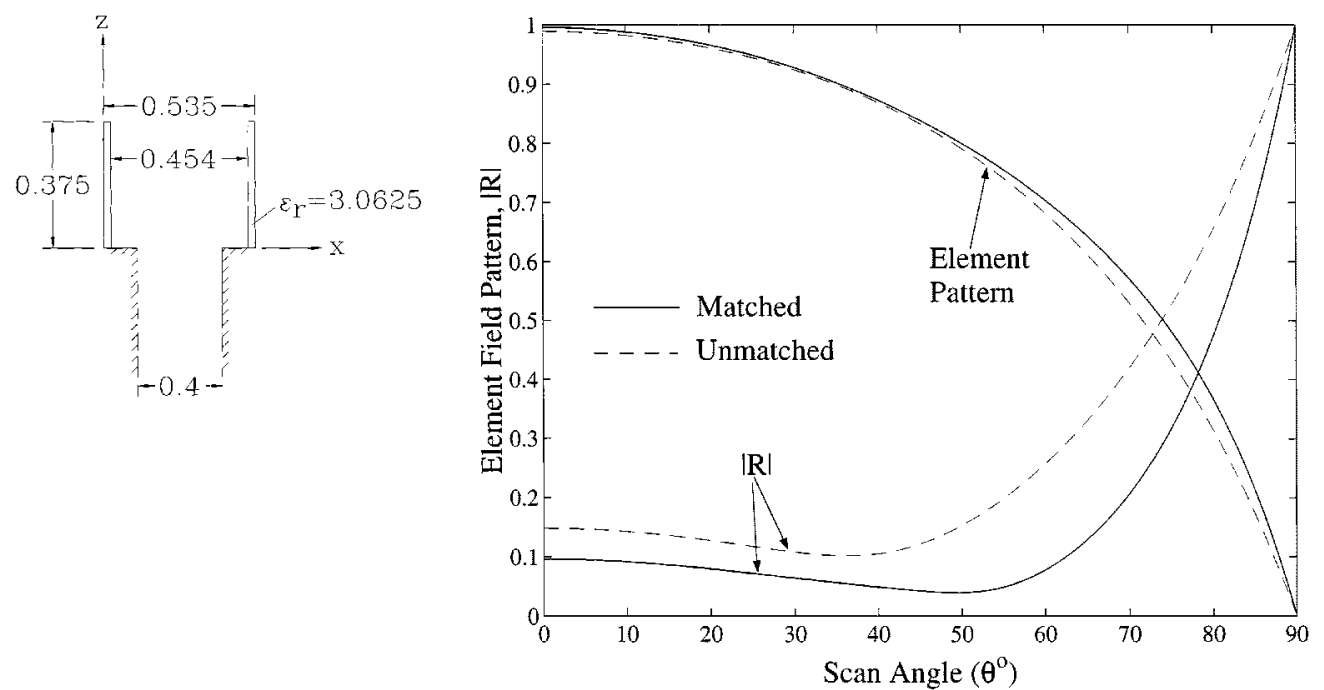

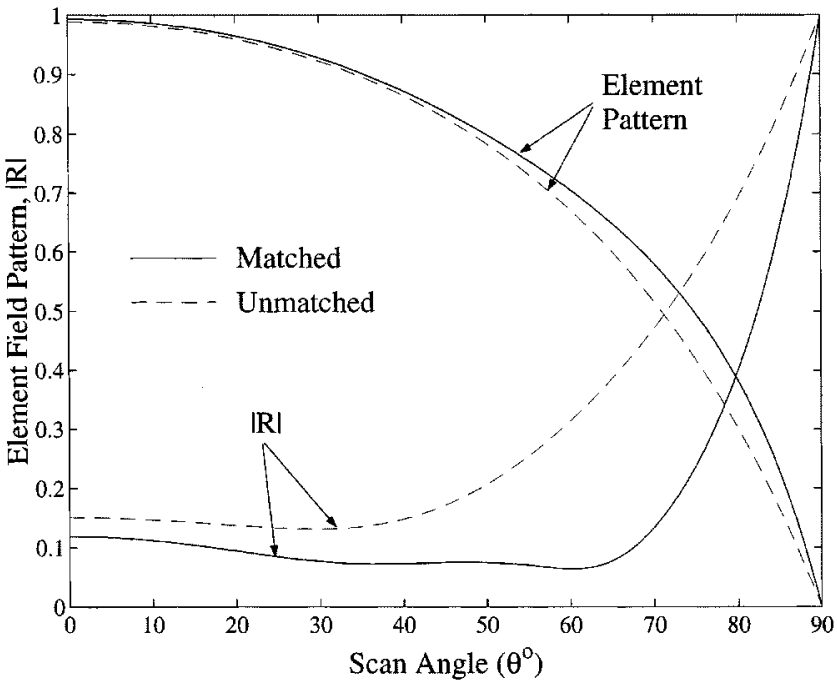

(c) (b)

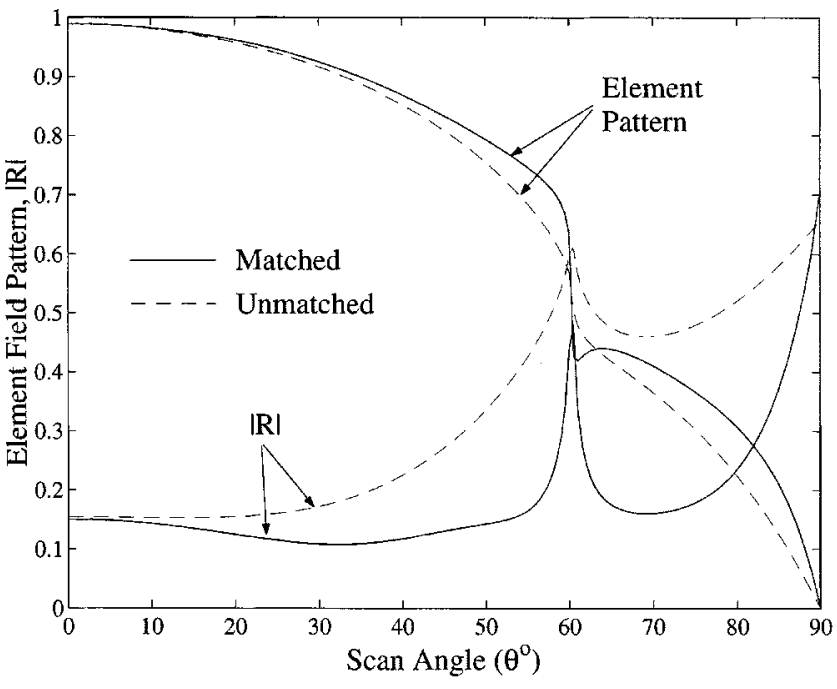

(d)

Fig. 6. (a) Geometry of an element with scan performance improved by dielectric protrusions. All dimensions are in free-space wavelengths at $3.0 \mathrm{GHz}$. (b)-(d) Comparison of the scanning characteristics for the dielectric-matched and unmatched element in Fig. 6(a) at: (b) 2.0, (c) 2.5, and (d) $3.0 \mathrm{GHz}$.

linked to undesired element pattern nulls. This means, as concluded in [3], that these types of configurations are not useful for wide-angle matching. However, as shown next, there are dielectric matching structures that may be suitable for this purpose. The particularly simple one considered here was obtained by choosing to move the protrusions into the regions of the unit cell where the fields are weaker, and the dielectric can modify the inter-element coupling and, thus, the element pattern in more subtle and controllable ways. Consider the element defined by the following dielectric distribution:

$$
\begin{aligned}
& \epsilon(x, z) \\
& \quad= \begin{cases}\epsilon_{0}, & x \in\left[x_{w}, x_{u}\right] ; z \in(-\infty, 0] \\
\epsilon_{r} \epsilon_{0}, & x \notin[a / 2-p(z), a / 2+p(z)] ; z \in\left[0, z_{e}\right] \\
\epsilon_{0}, & x \in[a / 2-p(z), a / 2+p(z)] ; z \in\left[0, z_{e}\right] .\end{cases}
\end{aligned}
$$

This configuration, shown in Fig. 6(a), is distinguished from (25) by the absence of a dielectric in the PPW and the placement of the protrusions close to the boundaries of the UCW. The formulas and definitions (27) apply with two minor changes, namely, the elimination of $\epsilon_{r}$ from the definition of the modal impedances in the PPW and the substitution $f_{m n}(z) \longrightarrow \delta_{m n}-$ $f_{m n}(z)$, where $\delta_{m n}$ is the Kronecker delta. The element dimensions at $3 \mathrm{GHz}$ are $a / \lambda_{0}=0.535, b / \lambda_{0}=0.4, \varepsilon_{r}=$ $3.0625, p(z) / \lambda_{0}=0.227, z_{e} / \lambda_{0}=0.375$. The reflection coefficient and element pattern for the unmatched and matched cases are compared in Fig. 6(b)-(d) for excitation frequencies of 2.0, 2.5 , and $3.0 \mathrm{GHz}$, respectively. It is clear that the dielectric inserts cause a significant extension in the scan range. Moreover, no undesired scan blindness is observed.

As a last example, the problem of designing a limited-scan element is considered. The unit-cell and waveguide dimensions, $a / \lambda_{0}=0.9, b / \lambda_{0}=0.3$, are the same as in [4, Fig. 8]. The dielectric distribution empirically designed to provide the desired element radiation pattern is shown in Fig. 7(a). The dielectric insert intrudes into both the PPW and UCW. The element pattern computed for this example is displayed in Fig. 7(b), alongside the pattern synthesized in [4, Fig. 8] for the same waveguide 


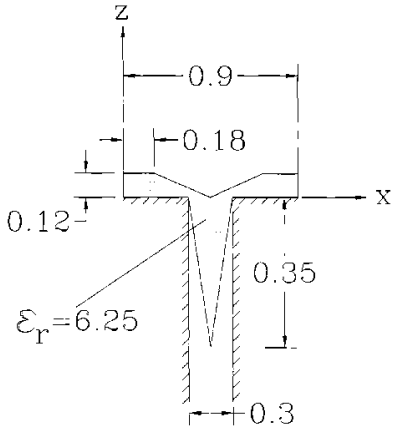

(a)

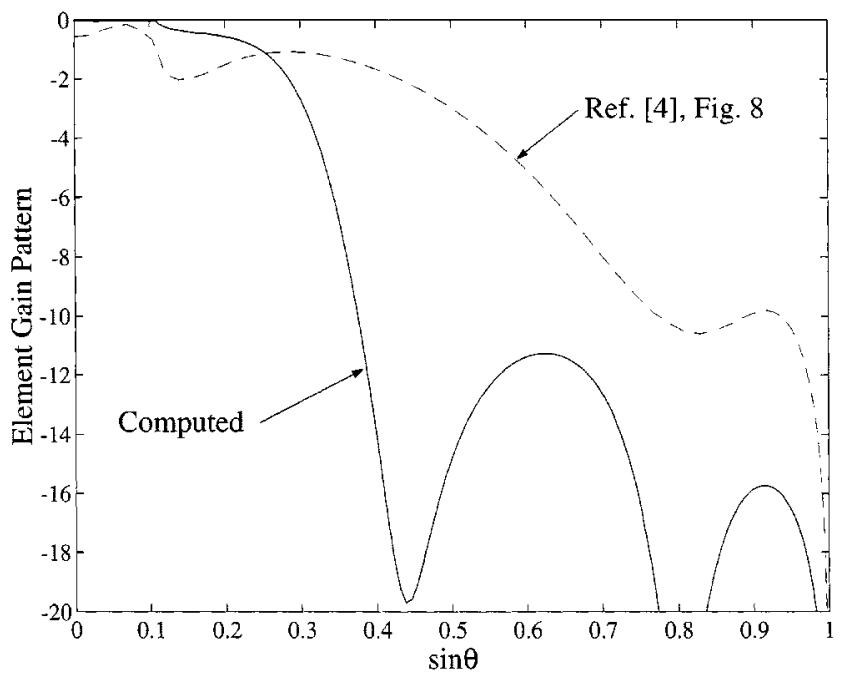

(b)

Fig. 7. (a) Geometry of an element suitable for limited scan. All dimensions are in free-space wavelengths. (b) Computed gain pattern for the element defined in Fig. 7(a). The gain pattern for an element with a shaped dielectric protrusion investigated in [4, Fig. 8] for comparison.

and unit-cell dimensions. A comparison of the two curves reveals that the design proposed here yields a significantly better limited-scan performance.

\section{CONCLUSIONS}

A novel method for analyzing waveguide phased arrays with general dielectric matching inserts in the aperture region has been presented and studied in this paper. The proposed technique represents a modification of the classical moment method solution of the waveguide array and, as such, preserves the key computational advantage of the latter, namely, the relatively small order of the final matrix problem. The generalization to arbitrary dielectric distributions comes at the cost of additional numerical processing (integration of an ODE set) required to calculate the fields in the regions containing the dielectric. For the problem at hand, this cost was not prohibitive. This can be attributed to the fact that only a few (PPW and UCW) modes are needed to approximate the fields excited by the entire domain basis functions chosen to represent the unknown aperture distribution.

Matching of waveguide elements by means of dielectric inserts has been deemed impractical, as previous studies have tied them to scan blindness. Using physical reasoning, a new matching configuration has been proposed here. Computed results indicate a significant scan range enhancement and no undesirable effects. Generalization of the analysis to allow for inhomogeneous dielectrics in the feeding waveguide has also led to a new way for synthesizing element patterns for limited-scan applications.

\section{ACKNOWLEDGMENT}

The author wishes to acknowledge the support of Dr. J. Franck, Air Force Office of Scientific Research, Washington, DC, and P. Gibber, Air Force Office of Scientific Research, Washington, DC, for their help and guidance, Prof. F. Gardiol, LEMA, Ecole Polytechnique Federale de Lausanne, Lausanne, Switzerland, and Prof. J. Mosig, LEMA, Ecole Polytechnique Federale de Lausanne, Lausanne, Switzerland, and their colleagues for generously sharing their time and facilities.

\section{REFERENCES}

[1] R. C. Hansen, Significant Phased Array Papers. Norwood, MA: Artech House, 1973.

[2] L. R. Lewis and A. Hessel, "Propagation characteristics of periodic arrays of dielectric slabs," IEEE Trans. Microwave Theory Tech., vol. MTT-19, pp. 276-286, Mar. 1971.

[3] L. R. Lewis, A. Hessel, and G. H. Knittel, "Performance of protrudingdielectric waveguide element in a phased array," IEEE Trans. Antennas Propagat., vol. AP-20, pp. 712-722, June 1972.

[4] S. P. Skobelev and L. L. Mukhamedov, "Analysis of waveguide antenna arrays with protruding dielectric elements," IEEE Trans. Antennas Propagat., vol. 41, pp. 574-581, May 1993.

[5] R. F. Harrington, Field Computation by Moment Method. New York: Macmillan, 1968.

[6] N. Amitay, V. Galindo, and C. P. Wu, Theory and Analysis of Phased Array Antennas. New York: Wiley, 1972.

[7] S. A. Schelkunoff, "Generalized telegraphist's equations for waveguides,” Bell Syst. Tech. J., pp. 784-801, July 1952.

[8] B. Z. Katzenelenbaum, Theory of Perturbed Waveguides with Slowly Varying Parameters (in Russian). Moscow, Russia: Akademiya Nauk SSSR, 1961.

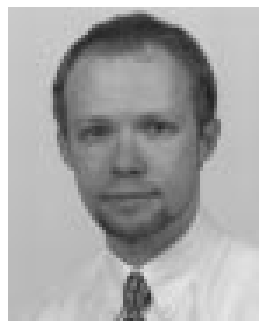

Marat Davidovitz (S'81-M'81-SM'95) received the B.S. (highest honors) and M.S. degrees in electrical engineering from the University of Illinois at Chicago, in 1981 and 1983, respectively, and the Ph.D. degree in electrical engineering from the University of Illinois at Urbana-Champaign, in 1987. From 1987 to 1988, he was an Alexander von Humboldt Post-Doctoral Fellow at DLR, Oberpfaffenhofen, Germany. From 1988 to 1993, he was with the Department of Electrical Engineering, University of Minnesota, Minneapolis-St. Paul. Since 1994, he is an Electronics Engineer at the Air Force Research Laboratory, Hanscom $\mathrm{AFB}, \mathrm{MA}$. 